\title{
LABORATORY DETERMINATION OF THE INFRARED BAND STRENGTHS OF PYRENE FROZEN IN WATER ICE: IMPLICATIONS FOR THE COMPOSITION OF INTERSTELLAR ICES
}

\author{
E. E. Hardegree-UllmaN ${ }^{1,2}$, M. S. Gudipati ${ }^{3,4}$, A. C. A. BoogerT ${ }^{2,5}$, H. Lignell ${ }^{6,7}$, \\ L. J. Allamandola ${ }^{8}$, K. R. Stapelfeldt ${ }^{9}$, and M. Werner ${ }^{3}$ \\ ${ }^{1}$ New York Center for Astrobiology and Department of Physics, Applied Physics, and Astronomy, Rensselaer \\ Polytechnic Institute, 110 8th Street, Troy, NY 12180, USA; hardee@ rpi.edu \\ ${ }^{2}$ Infrared Processing and Analysis Center, Mail Code 100-22, California Institute of Technology, Pasadena, CA 91125, USA \\ ${ }^{3}$ Jet Propulsion Laboratory, California Institute of Technology, 4800 Oak Grove Drive, Pasadena, CA 91109, USA \\ ${ }^{4}$ IPST, University of Maryland, College Park, MD 20742, USA; gudipati@jpl.nasa.gov \\ ${ }^{5}$ SOFIA Science Center, USRA, NASA Ames Research Center, M.S. N232-12, Moffett Field, CA 94035, USA \\ ${ }^{6}$ Department of Chemistry, University of California Irvine, Irvine, CA 92697-2025, USA \\ ${ }^{7}$ Division of Chemistry and Chemical Engineering, California Institute of Technology, Pasadena, CA 91125, USA \\ ${ }^{8}$ Space Science Division, Mail Stop 245-6, NASA Ames Research Center, Moffett Field, CA 94035, USA \\ ${ }^{9}$ NASA Goddard Space Flight Center, Exoplanets and Stellar Astrophysics Laboratory, Code 667, Greenbelt, MD 20771, USA \\ Received 2014 January 1; accepted 2014 February 14; published 2014 March 18
}

\begin{abstract}
Broad infrared emission features (e.g., at 3.3, 6.2, 7.7, 8.6, and $11.3 \mu \mathrm{m}$ ) from the gas phase interstellar medium have long been attributed to polycyclic aromatic hydrocarbons (PAHs). A significant portion (10\%-20\%) of the Milky Way's carbon reservoir is locked in PAH molecules, which makes their characterization integral to our understanding of astrochemistry. In molecular clouds and the dense envelopes and disks of young stellar objects (YSOs), PAHs are expected to be frozen in the icy mantles of dust grains where they should reveal themselves through infrared absorption. To facilitate the search for frozen interstellar PAHs, laboratory experiments were conducted to determine the positions and strengths of the bands of pyrene mixed with $\mathrm{H}_{2} \mathrm{O}$ and $\mathrm{D}_{2} \mathrm{O}$ ices. The $\mathrm{D}_{2} \mathrm{O}$ mixtures are used to measure pyrene bands that are masked by the strong bands of $\mathrm{H}_{2} \mathrm{O}$, leading to the first laboratory determination of the band strength for the $\mathrm{CH}$ stretching mode of pyrene in water ice near $3.25 \mu \mathrm{m}$. Our infrared band strengths were normalized to experimentally determined ultraviolet band strengths, and we find that they are generally $\sim 50 \%$ larger than those reported by Bouwman et al. based on theoretical strengths. These improved band strengths were used to reexamine YSO spectra published by Boogert et al. to estimate the contribution of frozen PAHs to absorption in the 5-8 $\mu \mathrm{m}$ spectral region, taking into account the strength of the $3.25 \mu \mathrm{m} \mathrm{CH}$ stretching mode. It is found that frozen neutral PAHs contain 5\%-9\% of the cosmic carbon budget and account for 2\%-9\% of the unidentified absorption in the 5-8 $\mu \mathrm{m}$ region.
\end{abstract}

Key words: astrochemistry - infrared: ISM - ISM: abundances - methods: laboratory: molecular - stars: protostars

Online-only material: color figures

\section{INTRODUCTION}

Unidentified infrared bands discovered almost $40 \mathrm{yr}$ ago in the interstellar medium (ISM) are now attributed to the $\mathrm{CH}$ and CC vibrational modes of polycyclic aromatic hydrocarbons (PAHs). They are estimated to account for $10 \%-20 \%$ of the total carbon reservoir in the ISM (Peeters 2011). Absorption features at 3.25 (Sellgren et al. 1994; Brooke et al. 1996, 1999), 6.2 (Keane et al. 2001), and $11.3 \mu \mathrm{m}$ (Bregman et al. 2000) have been attributed to PAHs in a handful of young stellar object (YSO) spectra, while PAH emission bands are weak or absent toward embedded YSOs (Geers et al. 2009). In molecular clouds and in the disks and envelopes of embedded YSOs, PAHs, along with other gas phase species, are expected to freeze out on the icy mantles of dust grains. In the dense ISM, ice mantles are important sites for prebiotic chemistry, where the long residence times of condensed molecules give them the best opportunity for interaction. Indeed, laboratory experiments have shown the ease of producing biomolecules via irradiation and heating of interstellar ice analogs (e.g., Bernstein et al. 2002; Muñoz Caro et al. 2002; Kaiser et al. 2013). Much work has been done to understand PAH chemistry in ice matrices (e.g., Gudipati \& Allamandola 2003; Gudipati 2004; Gudipati \& Allamandola 2004). In particular, PAHs frozen in amorphous $\mathrm{H}_{2} \mathrm{O}$ ice ionize quickly when irradiated by UV light. Larger PAHs remain stable up to $120 \mathrm{~K}$, at which point the ice crystallizes and PAHs begin to react with the ice matrix itself (Gudipati \& Allamandola 2006; Allamandola 2011), while smaller PAH ions react at lower temperatures to form hydrogenated and oxygenated complex molecules (Gudipati \& Yang 2012).

Significant attention has also been given toward the task of quantifying PAHs in the ISM. An extensive online database at www.astrochem.org (Bauschlicher et al. 2010; Boersma et al. 2014) contains both theoretical and experimental spectra of PAHs matrix isolated in argon. These spectra greatly enhance our understanding of PAH signatures, but spectra of PAHs embedded in interstellar ice analogs are necessary to truly constrain PAH abundances within the dense ISM. Experiments have been carried out to determine band strengths for both neutral (Bernstein et al. 2005; Bouwman et al. 2011) and ionized (Bernstein et al. 2007) pyrene frozen in water ice, but the absolute infrared (IR) band strengths reported in these cases were normalized to theoretically calculated band strengths. Sometimes, the region of interest reported was constrained between 1650 to $1000 \mathrm{~cm}^{-1}(6-10 \mu \mathrm{m})$ because the $\mathrm{H}_{2} \mathrm{O}$ ice absorption there is less significant and more linear than in other regions and none of these studies address the $\mathrm{CH}$ stretching mode at $3.25 \mu \mathrm{m}$, which is most easily accessible with groundbased telescopes. Band strengths for naphthalene frozen in water ice have been reported as well (Sandford et al. 2004). 


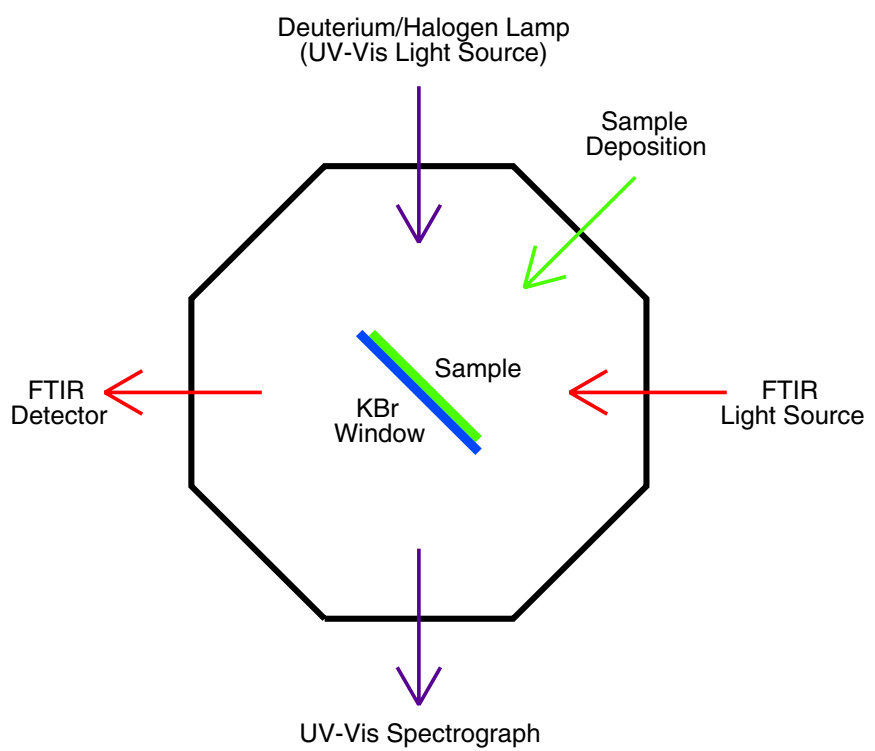

Figure 1. Experimental setup. The pressure in the chamber was kept at $\sim 10^{-7}$ mbar, and the temperature of the $\mathrm{KBr}$ window was kept at $30 \mathrm{~K}$. The frozen pyrene sample was generated by passing water vapor over sublimating pyrene crystals. This gaseous mixture then froze onto the $\mathrm{KBr}$ window. Transmission spectra were recorded using FTIR and UV-Vis spectrometers.

(A color version of this figure is available in the online journal.)

The aim of our work is to improve upon previous band strength estimates by recording PAH spectra in both infrared and ultraviolet-visible (UV-Vis) bands, then normalizing the results to previous, direct measurements of UV absolute band strengths (Berlman 1971; Dixon et al. 2005). This paper reports our results for pyrene $\left(\mathrm{C}_{16} \mathrm{H}_{10}\right)$ frozen in either $\mathrm{H}_{2} \mathrm{O}$ or $\mathrm{D}_{2} \mathrm{O}$ ice. Pyrene was chosen as a representative $\mathrm{PAH}$ molecule because it has been widely studied in the laboratory and a significant amount of laboratory data exists. The pyrene radical cation is proposed to be the carrier of some of the diffuse interstellar bands (DIBs; Salama \& Allamandola 1992). Additionally, pyrene is the smallest available $\mathrm{PAH}$ that is convenient to handle but at the same time compact with a $\mathrm{C} / \mathrm{H}$ ratio of 1.9 which is closer to the larger $\mathrm{PAH} \mathrm{C} / \mathrm{H}$ ratio expected in the ISM (see Section 4.3). We included $\mathrm{D}_{2} \mathrm{O}$ in our experiments because its absorption features are redshifted compared to those in $\mathrm{H}_{2} \mathrm{O}$, allowing easier measurement and detection of some pyrene bands which would normally be drowned out by $\mathrm{H}_{2} \mathrm{O}$ features. With revised band strengths in hand, we attempted to identify the previously reported $3.25 \mu \mathrm{m}$ band (Sellgren et al. 1994; Brooke et al. 1996, 1999) in the set of YSO spectra published by Boogert et al. (2008) and, where found, constrained PAH column densities and the contribution of PAH absorption to the 5-8 $\mu \mathrm{m}$ absorption region. Section 2 describes the experimental setup for obtaining spectral measurements, while Section 3 details our procedure for calculating absolute band strengths. Section 4 describes the astrophysical implications for quantifying PAHs in the dense ISM. Section 5 summarizes our findings and suggests possible trajectories for further expansion of this work.

\section{EXPERIMENTAL SETUP}

The setup for our laboratory experiment was very similar to that described in Barnett et al. (2012); a schematic is shown in Figure 1. In summary, the chamber for this experiment was cooled to $30 \mathrm{~K}$, at which temperature the base pressure in the chamber was in the high $10^{-9}$ to low $10^{-8}$ mbar. Water vapor was passed over pyrene crystals, which were heated to $\sim 55^{\circ} \mathrm{C}$ for sublimation under vacuum. This mixture of sublimated pyrene and water vapor was directed into the chamber, where it subsequently froze onto a potassium bromide $(\mathrm{KBr})$ window. During the ice deposition, pressure in the chamber was typically around $10^{-7}$ to $10^{-6}$ mbar due to the water vapor lead into the chamber. Under these conditions, the pyrene concentration was less than three percent in all samples, ensuring that pyrene molecules were adequately isolated from each other within the ice matrix. The pyrene concentration was further monitored and controlled as necessary during the experiments by measuring the ice film thickness, adjusting the pyrene sublimation temperature, and monitoring the IR and UV-Vis absorption features. Ice film deposition rates were approximately $1 \mu \mathrm{m}$ per hour. Transmission spectra through samples ranging in thickness from a few hundred nanometers to $5 \mu \mathrm{m}$ were collected via Fourier transform infrared (FTIR) spectroscopy and a UV-Vis spectrograph using a deuterium/halogen lamp as a continuum source. The spectral resolution and spectral coverage of the FTIR spectrometer were $1 \mathrm{~cm}^{-1}$ and $8000-400 \mathrm{~cm}^{-1}$, respectively. The UV-Vis spectrometer had a resolution of $\sim 0.75 \mathrm{~nm}$ in the 210-1100 $\mathrm{nm}$ wavelength range. Each instrument was connected to a separate port of the octagonal chamber, allowing IR and UV spectra to be collected consecutively. The optical window was oriented in such a way that it was as close to perpendicular as possible to the deposition side, at the same time allowing both FTIR and UV-Vis spectral measurements without rotating the cryostat on which the optical window was mounted. This configuration gave us highly reproducible spectra as we grew the ice slowly on the optical window. Roughly half the experiments were conducted with $\mathrm{H}_{2} \mathrm{O}$ and half with $\mathrm{D}_{2} \mathrm{O}$ in order to recover better measurements in areas with significant ice absorption. During $\mathrm{D}_{2} \mathrm{O}$ matrix experiments, even though we passivated the chamber with $\mathrm{D}_{2} \mathrm{O}$, there was still obvious contamination of $\mathrm{H}_{2} \mathrm{O}$ ice features in the spectra at times, necessitating great care when interpreting band strengths in regions of overlap between ice features.

Atmospheric water vapor in the path of the FTIR instrument and the detector was minimized by passing gaseous nitrogen through the enclosed regions. However, there were several openings, which were necessary to optimize the instrumentation, that could not be completely sealed off. As a consequence, vapor-phase water absorption is always present in our infrared spectra. Fortunately, due to their characteristic absorption, we have routinely subtracted the water vapor absorption from the ice spectra, as will be discussed in the next section. Water vapor spectra were derived from measuring the spectra of the experimental setup at room temperature before and after passing dry nitrogen gas at a very high flow rate (10 times more than the normal flow rate of $\sim 20 \mathrm{~L} /$ minute), eliminating a significant amount of water vapor over a short period of time.

\section{ANALYSIS}

Absolute IR band strengths of ices are difficult to calculate directly from laboratory experiments because column densities require a determination of the sample density, thickness, and mixing ratio. In order to determine absolute IR band strengths of pyrene frozen in water ice, we correlated our measured IR pyrene bands to the first electronic transition of pyrene. The 
absolute band strength of this UV band is easily calculated from previously published data.

\subsection{UV Band Strength And Integrated Absorption}

The molar absorptivity, $\epsilon\left(\mathrm{M}^{-1} \mathrm{~cm}^{-1}\right)$, of the first electronic transition of pyrene (in cyclohexane) was measured by Berlman (1971). This transition occurs in the UV frequency range of $\sim 35,000-28,800 \mathrm{~cm}^{-1}(286-347 \mathrm{~nm})$. Dixon et al. (2005) duplicated the experiment and published their results in the online database PhotochemCAD. ${ }^{10}$ Their results were normalized at the peak of the first electronic transition to the value $\epsilon=54,000 \mathrm{M}^{-1} \mathrm{~cm}^{-1}$ listed by Berlman (1971), which agrees with the results found by Thony \& Rossi (1997) for gas-phase pyrene at $150 \mathrm{~K}$. From these measurements, we directly calculated the integrated band strength of the first electronic transition of pyrene using Equations (1)-(4). Although these experiments measured pyrene in cyclohexane rather than in ice, the total oscillator strength of any given electronic transition remains constant (a molecular property), irrespective of the solvents used, unless strong electronic interactions between the solvent and the PAH molecule occurs that could change electronic and vibrational transitions significantly. This is not the case in cyclohexane which is a non-polar solvent. Amorphous ices behave like non-polar solvents with electronic polarizability similar to argon matrix, so strong interactions between pyrene and amorphous water ice are negligible (Gudipati 2004). Therefore, the assumption that the integrated UV band strength for the first electronic transition of pyrene is equivalent in amorphous water ice and cyclohexane is valid.

Integrated band strengths, $A$, are calculated using

$$
A=\frac{\int_{x_{1}}^{x_{2}} \tau(x) d x}{N}=\frac{2.303 \int_{x_{1}}^{x_{2}} a(x) d x}{N},
$$

where $\tau=\ln \left(I_{0} / I\right)$ is the optical depth of the feature, $x$ is the wavenumber $\left(1 / \lambda, \mathrm{cm}^{-1}\right), N$ is the column density $\left(\mathrm{cm}^{-2}\right)$ of the material producing the feature, and $a=\log _{10}\left(I_{0} / I\right)$ stands for absorbance, which is the preferred unit of experimental scientists.

After some manipulation, the Beer-Lambert law (2) can be substituted into (1) to determine the band strength directly from molar absorptivity. In Equations (2)-(4), $c$ is molar concentration $\left(1 \mathrm{M}=0.001 \mathrm{~mol} \mathrm{~cm}^{-3}\right), l$ is path length $(\mathrm{cm})$, $n$ is number density $\left(\mathrm{cm}^{-3}\right)$, and $N_{A}$ is Avogadro's constant $\left(6.02 \times 10^{23} \mathrm{~mol}^{-1}\right)$.

$$
\begin{gathered}
a=\epsilon c l \\
N=n l=\left(0.001\left[\frac{\mathrm{mol} \mathrm{cm}^{-3}}{M}\right]\right) N_{A} c l \\
A=\frac{2.303 \int_{x_{1}}^{x_{2}} \epsilon(x) d x}{\left(0.001\left[\frac{\mathrm{mol} \mathrm{cm}^{-3}}{M}\right]\right) N_{A}} .
\end{gathered}
$$

Before performing any of the following analyses, raw spectra were smoothed using a fast Fourier transform (FFT) filter (Bowman 2006) with a window ${ }^{11}$ of $p=5$ in order not to

\footnotetext{
10 www.photochemcad.com

11 See http://wiki.originlab.com/ originla/wiki2/index.php?title= $\mathrm{X}$-Function:Smooth. Note, however, a typo in the equation listed there. The term $(1-t)^{2}$ in the last line of the equation should instead be $(n-t)^{2}$.
}

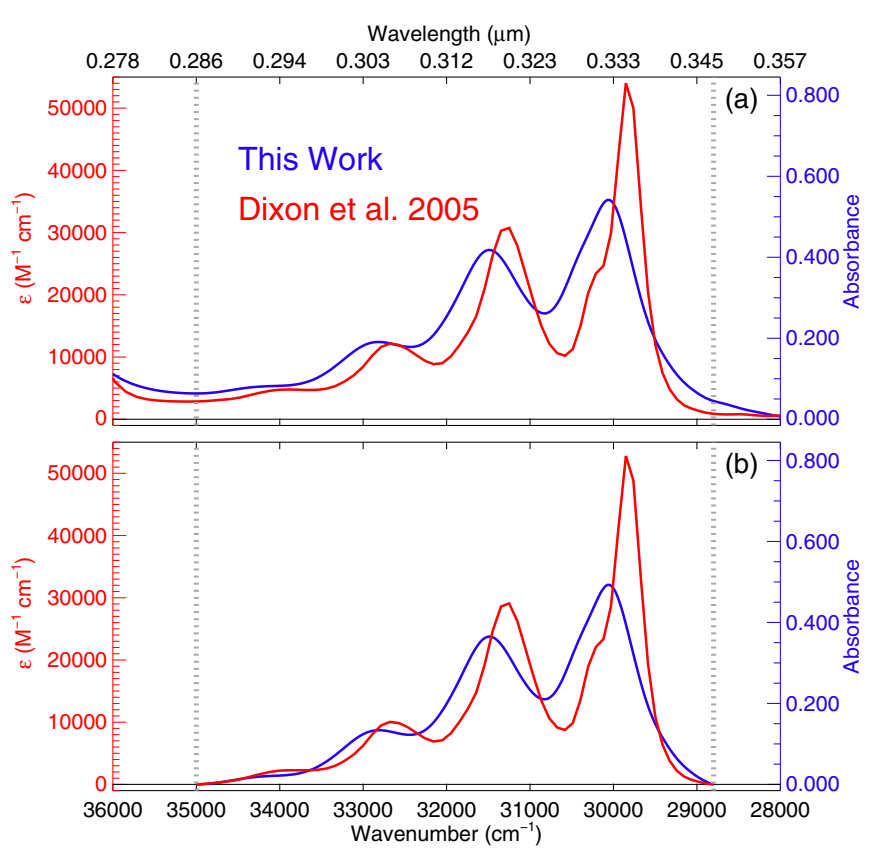

Figure 2. Panels (a) and (b) show intermediate steps for obtaining the absolute UV band strength for the first electronic transition of pyrene. An absorption spectrum selected from our sample of pyrene in $\mathrm{H}_{2} \mathrm{O}$ is shown in blue. The molar absorptivity spectrum of pyrene in cyclohexane, published by Dixon et al. (2005), is shown in red. Panel (a) shows original spectra, without baselining. Panel (b) shows the same spectra after subtracting linear baselines anchored at 35,000 and $28,800 \mathrm{~cm}^{-1}$ (dotted lines).

(A color version of this figure is available in the online journal.)

compromise actual signal. The integration of molar absorptivity was carried out after subtracting a local, linear baseline anchored at 35,000 and $28,800 \mathrm{~cm}^{-1}$ (see Figure 2). We calculated the UV band strength as $2.578 \times 10^{-13} \mathrm{~cm} /$ molecule.

The errors in molar extinction coefficients come not from the spectra, but determining the weight of the substance in solution (molarity) as well as whether the molecules form aggregates in solution or stay as monomers (Siu \& Duhamel 2008). A good comparative study on absorption of pyrene in various solvents is given in Ray et al. (2006), where significant changes in peak positions are seen. However, their integrated molar absorptivity remains essentially unaltered, because if the height decreases, the bandwidth increases. Hence, we estimate that the experimental band strengths derived for pyrene UV spectra are very accurate within a few percent uncertainty at the maximum.

Integrated absorbances of the first electronic transition of pyrene were calculated from our spectra using the same linear baseline as described above. Subtracting a local baseline is important for frozen pyrene, because absorption due to the ice matrix itself is significant. Errors on the integrated UV absorbances were determined by shifting the baseline by $3 \mathrm{~nm}$ in either direction. In the rest of this work, the pyrene column densities calculated from the UV spectra were used to derive band strengths for the infrared transitions.

\subsection{IR Integrated Absorption}

To determine the strength, peak position, and width of the IR pyrene features, contamination by $\mathrm{H}_{2} \mathrm{O}$ vapor and baseline curvatures (due to ice absorption) need to be removed first. Contamination by water vapor absorption is significant in many of the spectra from $\sim 2025$ to $1275 \mathrm{~cm}^{-1}$ (see Figure 3). Without removal, such contamination greatly affects the identification 


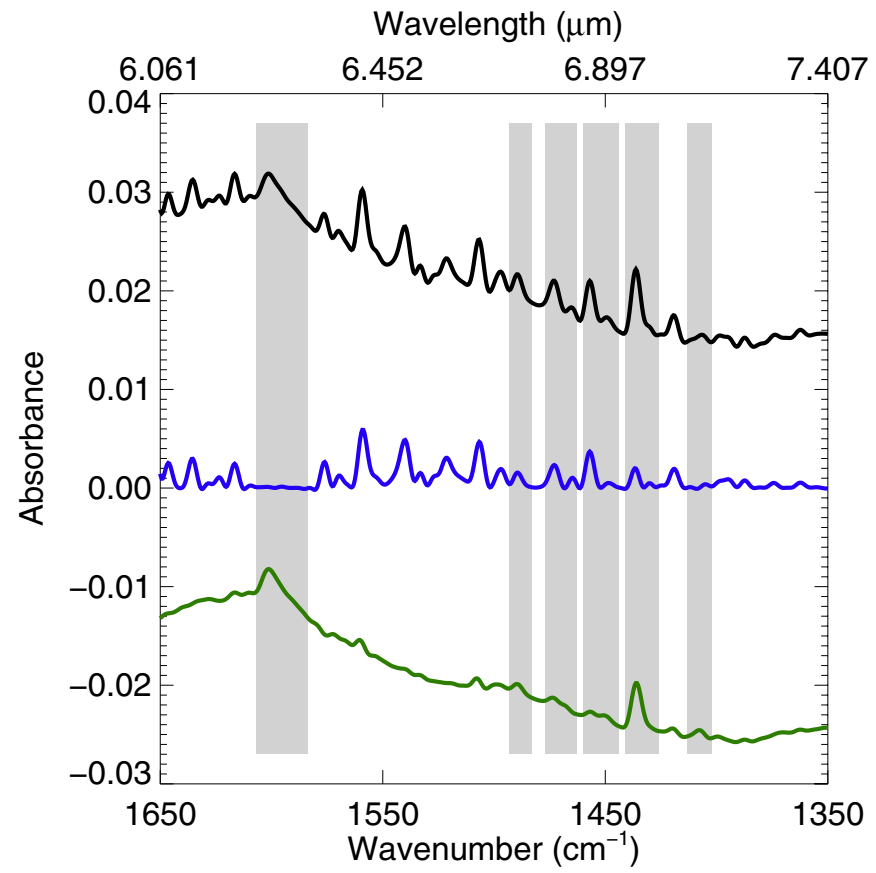

Figure 3. Typical example of water vapor subtraction. Regions where pyrene features exist are marked in gray. The original spectrum is shown in black, and the corrected spectrum (green) after subtraction of water vapor contamination (blue) is offset below.

(A color version of this figure is available in the online journal.)

and analysis of almost half of the IR pyrene bands. To obtain a water vapor template, we took the ratio of two background spectra. The water vapor residual was cleaned by the same FFT smoothing described above, and a local, low-order polynomial baseline was subtracted. The amplitudes of the five largest water vapor peaks unaffected by ice absorption were measured in each spectrum, and the water vapor template, scaled to those amplitudes, was then subtracted to remove the contamination.

To determine the integrated absorption of pyrene features in the IR, a global baseline to remove ice absorption was determined through implementation of the following method. First, pyrene features were masked (see Table 1), and the remaining spectrum was smoothed using Gaussian convolution.
Table 1

Masking Regions for Global Continuum

\begin{tabular}{lc}
\hline \hline Band Identification & $\begin{array}{c}\text { Masking Region } \\
\left(\mathrm{cm}^{-1}\right)\end{array}$ \\
\hline A & $3078-3032$ \\
B & $1607-1584$ \\
C & $1493-1483$ \\
D & $1477-1463$ \\
E & $1460-1444$ \\
F & $1441-1426$ \\
G & $1413-1402$ \\
H & $1321-1308$ \\
I & $1251-1235$ \\
J & $1194-1171$ \\
K & $1103-1091$ \\
L & $1072-1059$ \\
M & $864-837$ \\
N & $826-817$ \\
O & $767-746$ \\
P & $722-700$ \\
\hline
\end{tabular}

Notes. For this analysis, features were manually selected by visual inspection of the laboratory spectra using Bouwman et al. (2011) and the experimental results for pyrene in argon from www.astrochem.org (Bauschlicher et al. 2010; Boersma et al. 2014) as a guide. Although an additional feature at $1136 \mathrm{~cm}^{-1}$ was reported by Bouwman et al. (2011), it was not detected above $3 \sigma$ in our sample.

Then, the smoothed spectrum was interpolated across feature regions using a second-order polynomial. This global baseline was subtracted from the original spectrum to obtain a residual pyrene spectrum for which position, FWHM, and integrated area was determined for each band. Figure 4 provides an example of this method.

The error in the integrated area of IR bands was calculated by taking into account the standard deviation of absorption in the pyrene residuals. Since the noise can vary slightly with wavenumber, the standard deviation in absorption for a given feature was calculated within a region \pm 3 times the width of the masking region for that feature, ignoring any other pyrene features within these limits.
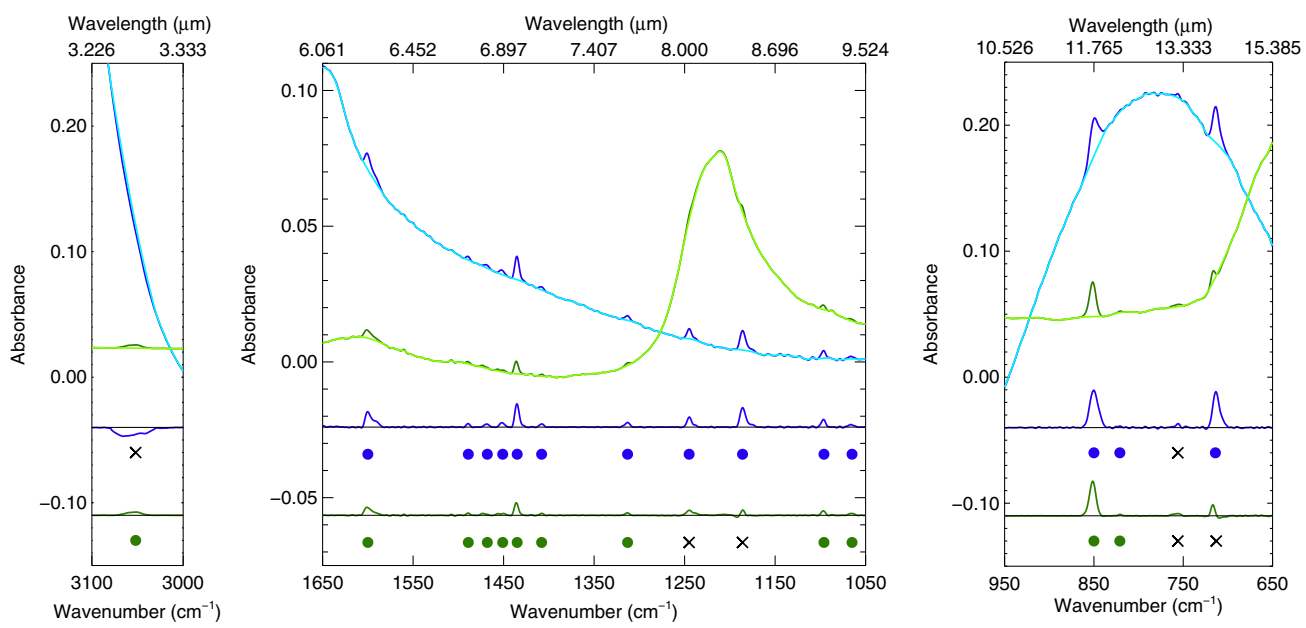

Figure 4. Typical examples of pyrene $/ \mathrm{H}_{2} \mathrm{O}$ and pyrene $/ \mathrm{D}_{2} \mathrm{O}$ spectra, after water vapor removal, are plotted with their baselines in the regions of pyrene absorption. $\mathrm{H}_{2} \mathrm{O}$ is in blue, and $\mathrm{D}_{2} \mathrm{O}$ is in green. The PAH residual spectra are offset below. Due to the way in which features were masked during baselining, not all PAH features were measured in each spectrum. Features that were measured are marked with a filled circle, and features that were ignored are marked with a cross. Note that the central panel has a much smaller $y$-axis scaling.

(A color version of this figure is available in the online journal.) 
Table 2

IR Pyrene Band Characteristics

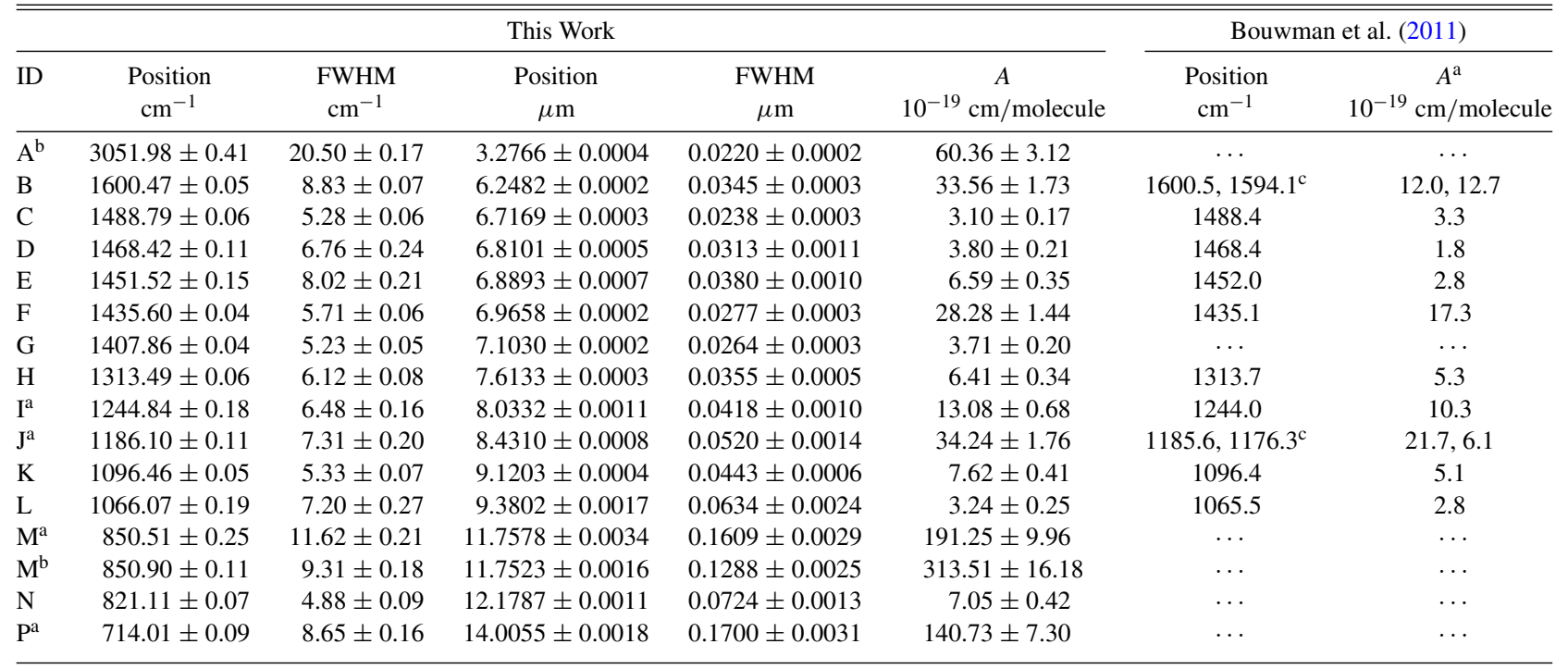

Notes. Some bands could only be measured in one ice matrix due to the inability to accurately determine the baseline ice absorption in the other ice species. Band $\mathrm{M}$ was measured separately in both $\mathrm{H}_{2} \mathrm{O}$ and $\mathrm{D}_{2} \mathrm{O}$ ice, as it appears to be $\sim 50 \%$ larger in a $\mathrm{D}_{2} \mathrm{O}$ ice matrix.

${ }^{\text {a }}$ Measured only in $\mathrm{H}_{2} \mathrm{O}$.

b Measured only in $\mathrm{D}_{2} \mathrm{O}$.

${ }^{c}$ Our laboratory spectra show one feature at this wavelength, whereas Bouwman et al. (2011) report two.

\subsection{Absolute IR Band Strengths}

The absolute IR band strengths were determined by correlating a strong infrared band with the calibrated UV band. This was challenging because the UV bands of pyrene tend to saturate fairly quickly while the weaker IR bands can take much longer to build up enough signal-to-noise for measurement. Figure 5 shows the correlation of the $1436 \mathrm{~cm}^{-1}$ band, as shown in Figure 4, with the UV band. This band is both fairly strong and relatively unaffected by ice absorption in either $\mathrm{H}_{2} \mathrm{O}$ or $\mathrm{D}_{2} \mathrm{O}$ ice. Since IR and UV spectra were taken consecutively rather than concurrently, IR measurements had to be interpolated to the observation times of the UV spectra. After determining the absolute band strength for the $1436 \mathrm{~cm}^{-1}$ band, the strengths of all bands were determined by correlating them with each other. Linear correlations were determined using the IDL program MPFITEXY (Williams et al. 2010), which utilizes the MPFIT package (Markwardt 2009). MPFIT is a curve fitting program that uses a robust, nonlinear least squares method. MPFITEXY was used to take into account $x$ and $y$ error bars and force the $y$ intercepts through the origin. Table 2 lists the position and FWHM of each band averaged over all spectra along with the final calculated band strengths.

\subsubsection{Comparison of Pyrene Band Strengths in $\mathrm{H}_{2} \mathrm{O}$ and $\mathrm{D}_{2} \mathrm{O}$ Ices}

Spectra were recorded for pyrene in $\mathrm{D}_{2} \mathrm{O}$ as well as in $\mathrm{H}_{2} \mathrm{O}$ ice in order to recover bands masked in laboratory spectra by the strong $\mathrm{H}_{2} \mathrm{O}$ features (e.g., the $3.25 \mu \mathrm{m} \mathrm{CH}$ stretching mode). This requires an assumption that $\mathrm{CH}$ stretching modes of pyrene behave identically whether the pyrene is frozen in $\mathrm{H}_{2} \mathrm{O}$ or $\mathrm{D}_{2} \mathrm{O}$ ice. Figure 6 demonstrates that this assumption is valid at least for the $\mathrm{CC}$ stretching and $\mathrm{CH}$ in plane bending modes of pyrene. It is not clear that this is the case for the $\mathrm{CH}$ out of plane bending modes. Unfortunately, beyond $10 \mu \mathrm{m}$, ice absorption in both $\mathrm{H}_{2} \mathrm{O}$ and $\mathrm{D}_{2} \mathrm{O}$ is quite strong and makes characterization of pyrene bands in that region more difficult.

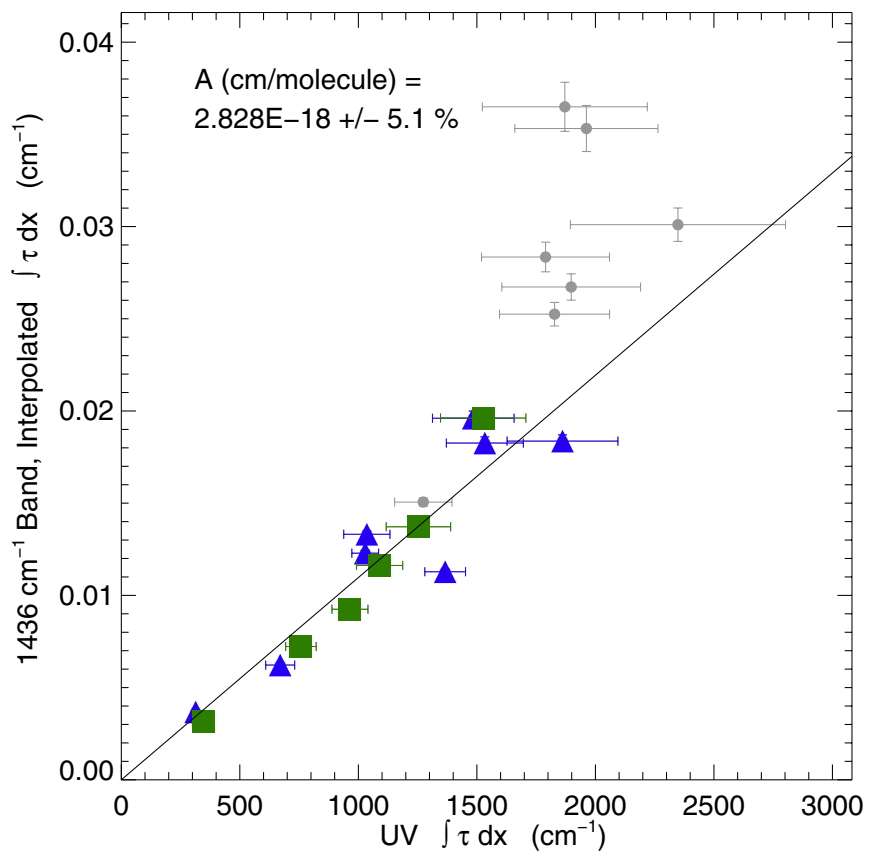

Figure 5. Correlation between the $1436 \mathrm{~cm}^{-1}$ IR band and the $35,000-28,800 \mathrm{~cm}^{-1} \mathrm{UV}$ band. Triangles show $\mathrm{H}_{2} \mathrm{O}$ samples and squares show $\mathrm{D}_{2} \mathrm{O}$ samples. Small circles show how the correlation breaks down as the UV band begins to saturate. Nonlinear growth of the UV features becomes significant above a peak absorbance of 0.8 (or $\tau$ of 1.84). Only unsaturated data points were used to determine the best fit linear correlation with the condition that it passes through the origin.

(A color version of this figure is available in the online journal.)

Local baselines could not be adequately determined for many of the pyrene bands beyond $10 \mu \mathrm{m}$, but the $850 \mathrm{~cm}^{-1}$ band appears to be measurable in both ice matrices. This was the only band in our analysis that was measured in both ices and gave different results depending on the ice matrix, as noted in Table 2. 


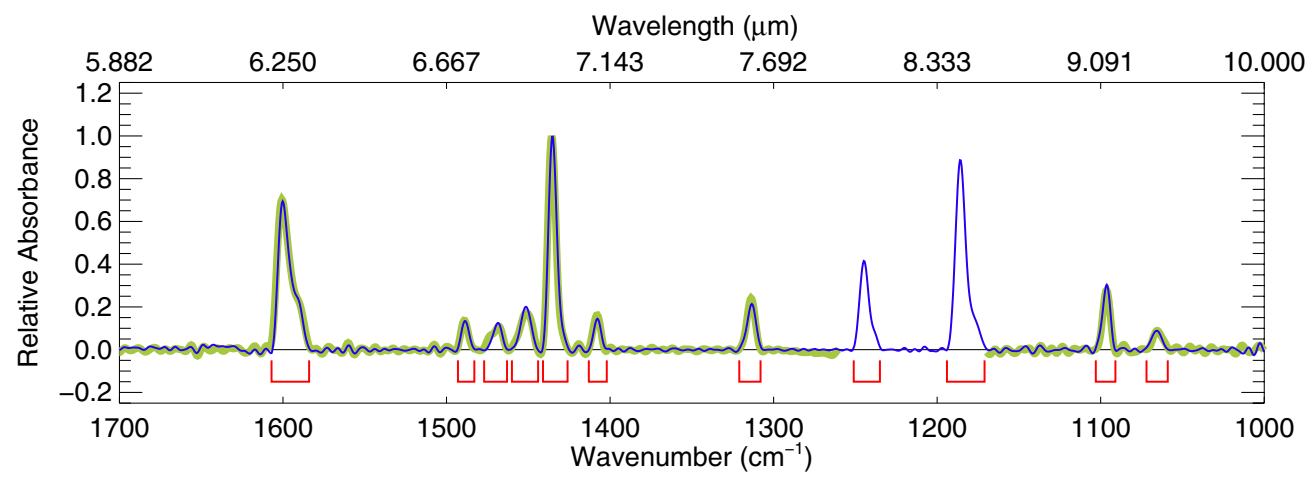

Figure 6. Comparison of the pyrene residual spectrum averaged across $\mathrm{H}_{2} \mathrm{O}$ (blue) and $\mathrm{D}_{2} \mathrm{O}$ (green) experiments. The average spectra were normalized at $1436 \mathrm{~cm}^{-1}$. Regions of obvious pyrene features are marked below in red. Some regions had to be excluded from the analysis due to inability to accurately determine baseline ice absorption, e.g., near $1200 \mathrm{~cm}^{-1}$ in $\mathrm{D}_{2} \mathrm{O}$ ice.

(A color version of this figure is available in the online journal.)
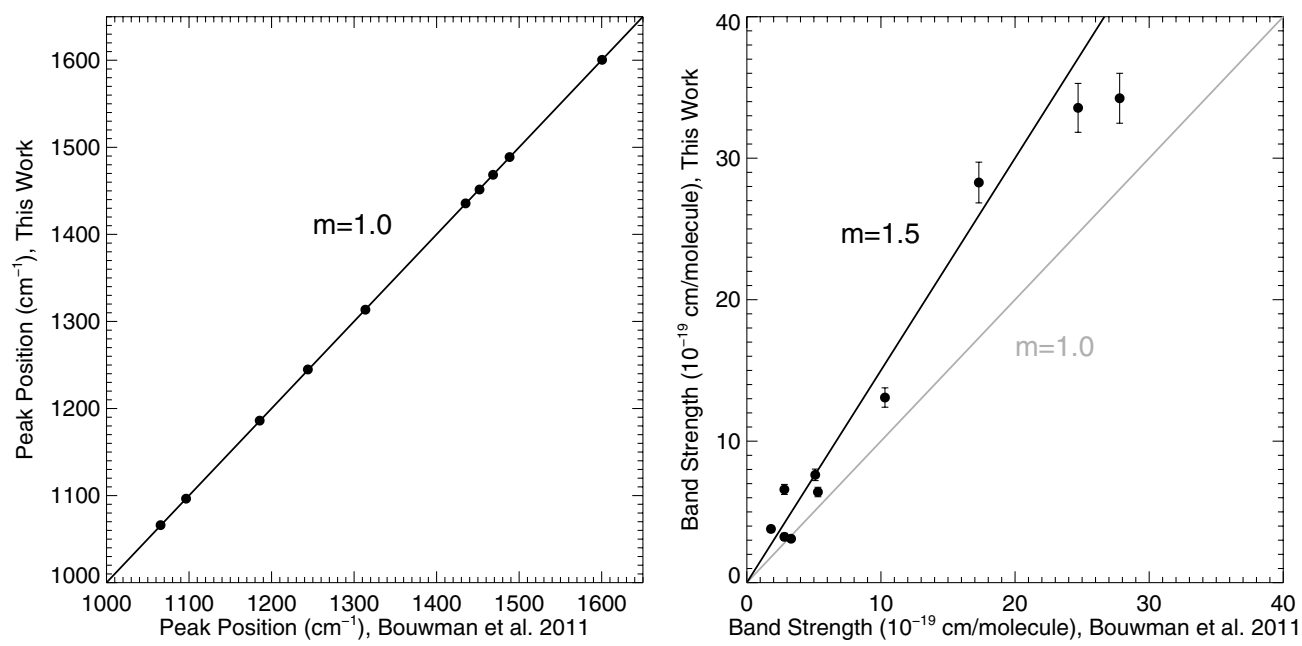

Figure 7. Peak position (left panel) and band strength (right panel) comparison between this work and that of Bouwman et al. (2011). The error bars along the $y$ axis in the left panel are smaller than the plot points. Our calculated band strengths are roughly 1.5 times those published by Bouwman et al. (2011).

\subsection{Comparison With Previous Laboratory Work}

Figure 7 compares our results with those published by Bouwman et al. (2011). While the positions of features measured in both works are essentially identical, the band strengths we report are $\sim 50 \%$ larger than those reported by Bouwman et al. (2011). Apparently, the theoretical band strength calculations used by Bouwman et al. (2011) consistently underestimate the values measured by our UV/IR correlation method.

\section{ASTROPHYSICAL IMPLICATIONS: PAH ABSORPTION FEATURES IN YSO SPECTRA AND THE CARBON BUDGET}

As illustrated here for pyrene, PAH IR spectra are characterized by a $\mathrm{CH}$ stretching feature near $3.25 \mu \mathrm{m}, \mathrm{CC}$ stretching and $\mathrm{CH}$ in plane bending modes between $5 \mu \mathrm{m}$ and $10 \mu \mathrm{m}$, and $\mathrm{CH}$ out of plane bending modes between roughly $10 \mu \mathrm{m}$ and $15 \mu \mathrm{m}$. The detection of a $3.25 \mu \mathrm{m}$ band, attributed to the $\mathrm{CH}$ stretching mode of PAHs, was reported for a handful of YSO spectra (Sellgren et al. 1994; Brooke et al. 1996, 1999). We looked for the presence of such a feature in the YSO spectra published by Boogert et al. (2008). For those spectra where a feature was detected, we attempted to quantify the PAH column density and the contribution of absorption by PAHs to the 5-8 $\mu \mathrm{m}$ region. Since we only have laboratory data for pyrene, the following measurements are reported as a function of the number of $\mathrm{CH}$ and $\mathrm{CC}$ bonds present. This of course requires the assumption that band strengths per bond remain relatively constant amongst different PAH species. Figure 8 shows the validity of this assumption. Here, experimentally determined band strengths per bond for neutral PAHs in argon from the www.astrochem.org database are shown as a function of the number of $\mathrm{CH}$ and $\mathrm{CC}$ bonds per molecule. For the $\mathrm{CH}$ stretching and $\mathrm{CC}$ stretching/ $\mathrm{CH}$ in plane bending modes, band strengths were integrated from 3200 to $2800 \mathrm{~cm}^{-1}$ and 2000 to $1250 \mathrm{~cm}^{-1}$, respectively.

\section{1. $3.25 \mu \mathrm{m}$ Feature}

We analyzed the $3.25 \mu \mathrm{m}$ absorption band in the YSO spectra published by Boogert et al. (2008). In summary, a total of 21 objects were observed with sufficient wavelength coverage to examine their spectra for a feature near $3.25 \mu \mathrm{m}$. Six of those objects were massive YSOs observed with the Short Wavelength Spectrometer on board the Infrared Space Observatory (also published by Gibb et al. 2004). The others were low mass YSOs observed in the $L$ band with ground-based instruments and longward of $5 \mu \mathrm{m}$ with the Spitzer Space Telescope.

Ground-based spectra were much noisier than the Infrared Space Observatory (ISO) spectra, so they were first smoothed with the boxcar technique using a window of five points. Only a subset of the $I S O$ spectra analyzed included flux density error bars, which were subsequently propagated to determine error 

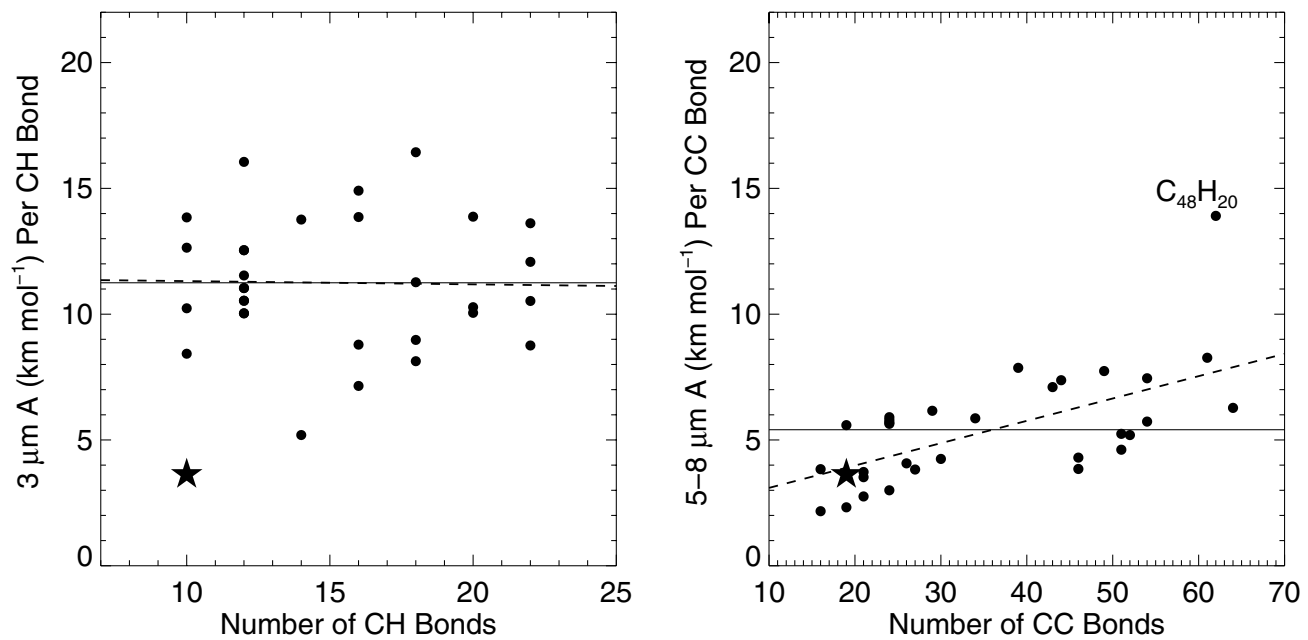

Figure 8. Experimentally measured band strengths for the $\mathrm{CH}$ stretching mode near $3.25 \mu \mathrm{m}$ (left) and the $\mathrm{CC}$ stretching/CH in plane bending modes between 5 to $8 \mu \mathrm{m}$ (right) from the www.astrochem.org database (Bauschlicher et al. 2010; Boersma et al. 2014) for neutral PAH species in argon (circles). Band strengths per bond remain fairly constant across reported PAH species, although the 5-8 $\mu \mathrm{m}$ band strengths do change mildly with a slope of $0.09 \mathrm{~km} \mathrm{~mol}^{-1}$ per CC bond per total number of $\mathrm{CC}$ bonds (excluding the outlier due to $\mathrm{C}_{48} \mathrm{H}_{20}$ ). Dashed lines show the best fitting correlations between band strengths per bond and number of bonds, while solid lines show the best fitting constant band strengths per bond. Stars mark the band strengths per bond for pyrene in water ice.

Table 3

Continuum Definition

\begin{tabular}{lcccc}
\hline \hline Object & $\begin{array}{c}\text { R.A. and Decl. } \\
(\mathrm{J} 2000)\end{array}$ & \multicolumn{3}{c}{$\begin{array}{c}\text { Continuum Regions } \\
(\mu \mathrm{m})\end{array}$} \\
\hline Mon R2 IRS 3 & $06^{\mathrm{h}} 07^{\mathrm{m}} 47^{\mathrm{s}} .80-06^{\circ} 22^{\prime} 55^{\prime \prime} .0$ & $3.15-3.20$ & $3.28-3.30$ & $3.70-3.80$ \\
S140 IRS 1 & $22^{\mathrm{h}} 19^{\mathrm{m}} 18^{\mathrm{s}} .17+63^{\circ} 18^{\prime} 47^{\prime \prime} \cdot 6$ & $3.15-3.20$ & $3.28-3.36$ & $3.70-3.80$ \\
GL 2136 & $18^{\mathrm{h}} 22^{\mathrm{m}} 26^{\mathrm{s}} .32-13^{\circ} 30^{\prime} 08^{\prime \prime} .2$ & $3.14-3.17$ & $3.25-3.33$ & $3.70-3.80$ \\
Ground-based spectra & $\cdots$ & $3.15-3.20$ & $3.28-3.36$ & $3.70-3.80$ \\
\hline
\end{tabular}

Table 4

$3.25 \mu \mathrm{m}$ Absorption Feature

\begin{tabular}{lcccc}
\hline \hline Object & $\lambda$ & \multicolumn{2}{c}{ FWHM } & \multirow{2}{*}{$\tau_{\max }$} \\
\cline { 3 - 4 } & $\mu \mathrm{m}$ & $\mu \mathrm{m}$ & $\mathrm{cm}^{-1}$ & \\
\hline Mon R2 IRS 3 & $3.244 \pm 0.002$ & $0.052 \pm 0.004$ & $49.3 \pm 3.4$ & $0.046 \pm 0.003$ \\
S140 IRS 1 & $3.247 \pm 0.001$ & $0.046 \pm 0.003$ & $44.1 \pm 3.1$ & $0.028 \pm 0.002$ \\
GL 2136 & $3.206 \pm 0.003$ & $0.051 \pm 0.006$ & $49.4 \pm 6.0$ & $0.163 \pm 0.018$ \\
Ground-based average & $3.258 \pm 0.004$ & $0.026 \pm 0.007$ & $24.1 \pm 6.9$ & $0.021 \pm 0.006$ \\
\hline
\end{tabular}

bars for the optical depth spectra. To determine error bars for the remaining optical depth spectra, a line was fit to a region $\pm 0.025 \mu \mathrm{m}$ on either side of each data point, and the standard deviation of that data set with respect to the linear fit was assigned as the error bar value for the relevant point.

For each spectrum, a low-order (third to fifth) polynomial was fit to a continuum region, defined in Table 3, depending on the absorption features of each spectrum. Next, the residual spectra were fit with up to five Gaussians, located at $\sim 3.25,3.32,3.36$, 3.47, and $3.53 \mu \mathrm{m}$, using MPFIT and MPFITFUN (Markwardt 2009). The $3.47 \mu \mathrm{m}$ feature, often seen in YSO spectra, has been attributed to solo hydrogens attached to "diamond"-like carbon clusters (Allamandola et al. 1992) or to ammonia hydrate (Dartois \& d'Hendecourt 2001). The remaining model features at $3.32,3.36$, and $3.53 \mu \mathrm{m}$ correspond to the expected locations of solid $\mathrm{CH}_{4}, \mathrm{C}_{2} \mathrm{H}_{6}$, and $\mathrm{CH}_{3} \mathrm{OH}$, respectively (e.g., Whittet 2003). In the ground-based spectra, the $3.32 \mu \mathrm{m}$ feature is heavily contaminated by telluric $\mathrm{CH}_{4}$ lines.

Of the ISO spectra, only three objects have a significant ( $>3 \sigma$ ) feature near $3.25 \mu \mathrm{m}$. Brooke et al. (1999), using groundbased spectra, listed previously reported measurements of the feature for Mon R2 IRS 3 and S140 IRS 1, but they excluded measurement of an apparent feature at $3.2 \mu \mathrm{m}$ for GL 2136 as they claim that its coincidence with the inflection point of crystalline ice absorption interferes with accurate measurement. These objects are shown in Figure 9, and the parameters of their model fits are listed in Table 4. The integrated optical depth measurement of the $3.25 \mu \mathrm{m}$ feature for Mon R2 IRS 3 is in good agreement with previous results by Sellgren et al. (1994, 1995), and our measurement for S140 IRS 1 is similar to that reported by Brooke et al. (1996) to within a factor of two.

For our ground-based YSO spectra, in order to increase the signal-to-noise ratio, all spectra were averaged to recover a global $3.25 \mu \mathrm{m}$ feature. The average, continuum subtracted spectrum, binned to $0.002 \mu \mathrm{m}$, is shown in Figure 10 on an optical depth scale. The best-fit Gaussian to the region of interest is located at $3.258 \pm 0.004 \mu \mathrm{m}$ with a maximum optical depth of $0.021 \pm 0.006$ and a FWHM of $24.13 \pm 6.93 \mathrm{~cm}^{-1}$ $(0.026 \pm 0.007 \mu \mathrm{m})$.

\subsection{5-8 $\mu \mathrm{m}$ Region}

Boogert et al. (2008) found that the 5-8 $\mu \mathrm{m}$ absorption regions in YSO spectra are due to at least five distinct components 

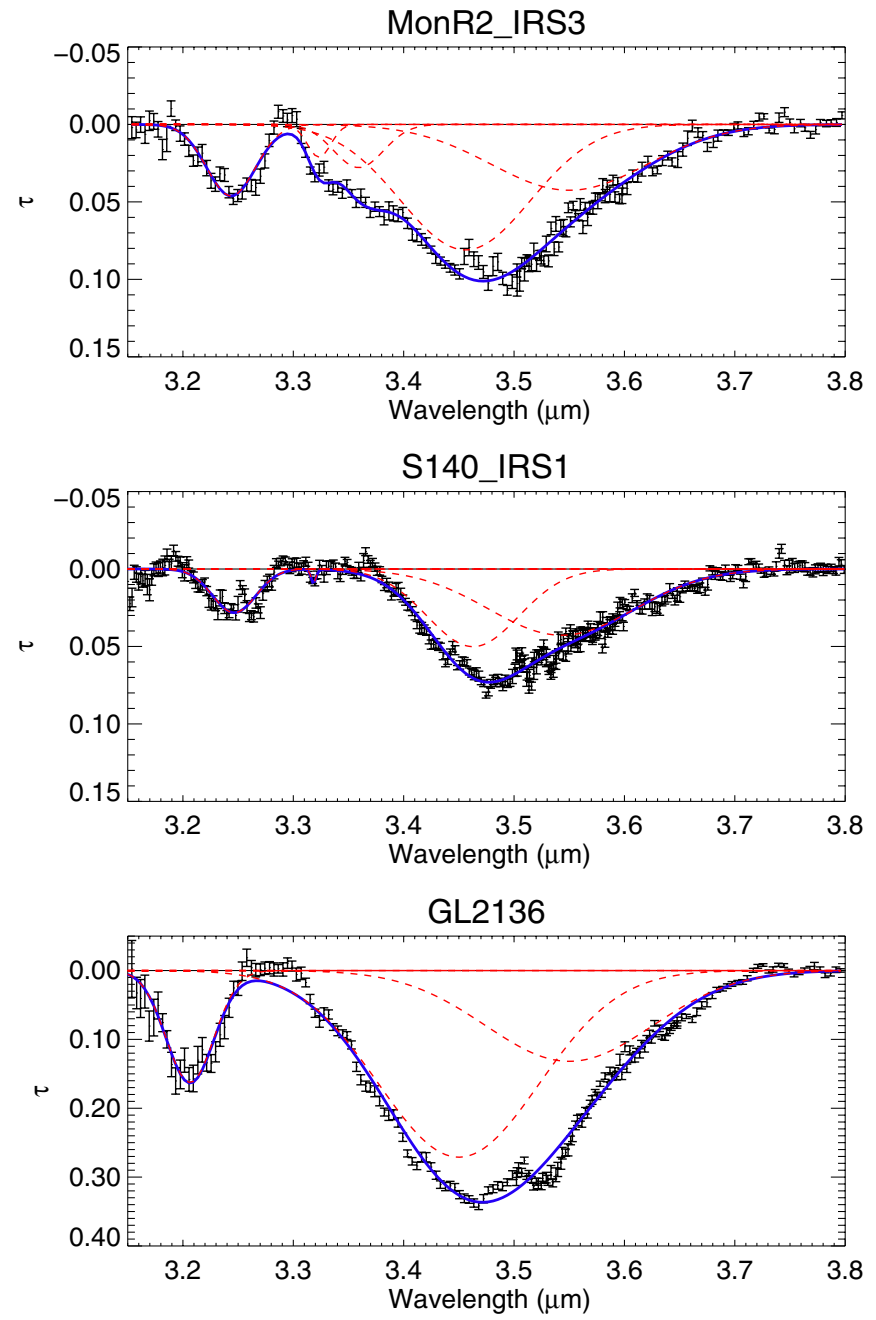

Figure 9. Optical depth spectral models after continuum subtraction. The model fits, composed of up to 5 Gaussians, are shown with solid blue lines. Each component of the model is shown separately with dashed red lines.

(A color version of this figure is available in the online journal.)

in addition to the bending mode of $\mathrm{H}_{2} \mathrm{O}$ ice, each of which likely have multiple carriers. Since PAHs exhibit a number of features in this same region due to $\mathrm{CC}$ stretching and $\mathrm{CH}$ in plane bending modes, we hypothesized that some of the absorption could be due to PAHs frozen in ice mantles.

Assuming that any $3.25 \mu \mathrm{m}$ feature present is due to neutral PAHs frozen in ice, we used our previous calculations of absolute band strengths for pyrene to constrain the potential $\mathrm{PAH}$ contribution to the $5-8 \mu \mathrm{m}$ absorption region $\left(f_{\mathrm{PAH}}\right.$, see Table 5), after subtraction of the $\mathrm{H}_{2} \mathrm{O}$ bending mode. Ours is only a first-order approximation since the PAHs present in the ISM are likely to consist of a mixture of many species, as clearly illustrated by Figure 11.

The average PAH molecule in the ISM contains 50 carbon atoms (Tielens 2008), rather than the 16 of pyrene. To account for this we assume that the band strengths per bond remain constant among different PAH species, although computational spectra predict the $\mathrm{CH}$ stretching band to increase for large PAHs (Bauschlicher et al. 2008), and we report contribution percentages in terms of the ratio of $\mathrm{CC}$ to $\mathrm{CH}$ bonds which characterizes the actual mixture of observed interstellar PAHs. For reference, that ratio is $\left(n_{\mathrm{CC}} / n_{\mathrm{CH}}\right)=1.9$ for pyrene. The simplest PAH, naphthalene $\left(\mathrm{C}_{10} \mathrm{H}_{8}\right)$, has a ratio of $\left(n_{\mathrm{CC}} / n_{\mathrm{CH}}\right)=$ 1.375 , and circumcoronene $\left(\mathrm{C}_{54} \mathrm{H}_{18}\right)$, the most compact and

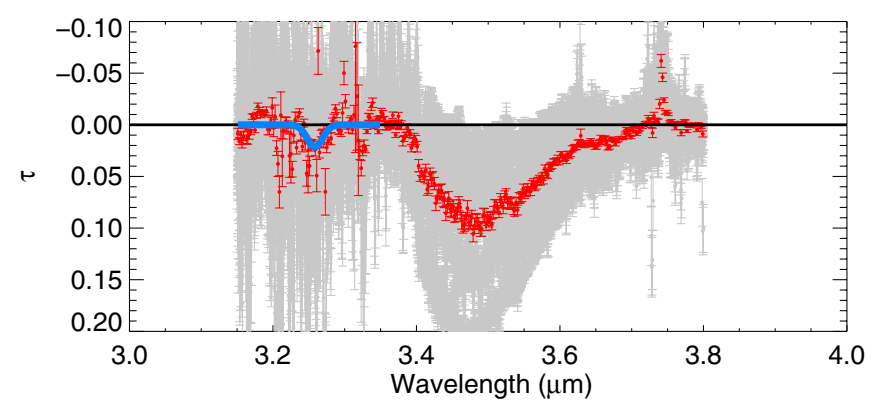

Figure 10. Average residual spectrum from ground-based observations, binned to $0.002 \mu \mathrm{m}$ (red). The individual residual spectra after continuum subtraction are all overplotted in gray. A Gaussian, fit to the prospective PAH feature, is shown in blue. The sharp feature near $3.32 \mu \mathrm{m}$ is due to telluric $\mathrm{CH}_{4}$.

(A color version of this figure is available in the online journal.)

radially symmetric $\mathrm{PAH}$ with $\sim 50$ carbon atoms, has a ratio of $\left(n_{\mathrm{CC}} / n_{\mathrm{CH}}\right)=4$. Equations (5)-(7) spell out how to calculate $f_{\mathrm{PAH}}$. In these equations, $\Delta x$ refers to the FWHM of a particular feature. Band strengths from bands $\mathrm{B}-\mathrm{H}$ in Table 2 were summed and divided by 19 (the number of $\mathrm{CC}$ bonds in pyrene) to obtain a band strength of $A_{\mathrm{CC}}=4.497( \pm 0.234) \times$ $10^{-19} \mathrm{~cm}$ per $\mathrm{CC}$ bond. The band strength of band $\mathrm{A}$ is $A_{\mathrm{CH}}=6.036( \pm 0.312) \times 10^{-19} \mathrm{~cm}$ per $\mathrm{CH}$ bond.

$$
\begin{gathered}
N(\mathrm{PAH})=\frac{\tau \Delta x_{3 \mu \mathrm{m}}}{A_{\mathrm{CH}} n_{\mathrm{CH}}}=\frac{\Sigma_{5-8 \mu \mathrm{m}} \tau \Delta x}{A_{\mathrm{CC}} n_{\mathrm{CC}}} \\
\Sigma_{5-8 \mu \mathrm{m}} \tau \Delta x=\tau \Delta x_{3 \mu \mathrm{m}} \frac{A_{\mathrm{CC}}}{A_{\mathrm{CH}}} \frac{n_{\mathrm{CC}}}{n_{\mathrm{CH}}} \\
f_{\mathrm{PAH}}=\frac{\Sigma_{5-8 \mu \mathrm{m}} \tau \Delta x}{\int \tau d x_{\mathrm{obs}}} \times 100 .
\end{gathered}
$$

\subsection{Carbon Budget}

Observations of PAH emission from the diffuse ISM indicate that $10 \%-20 \%$ of the total carbon is locked in PAH molecules (Peeters 2011). The column densities of $\mathrm{CH}$ bonds reported in Table 5 can be used to calculate the fraction of the carbon abundance locked up in PAHs in the ices around YSOs. Hydrogen column densities reported by Boogert et al. (2013) were converted to carbon column densities using $x(\mathrm{C})=$ $3.9 \times 10^{-4}$ (Tielens 2008). The number of carbon atoms locked in PAHs were calculated from $N(\mathrm{CH})$ assuming that the average $\mathrm{PAH}$ molecule contains $\sim 22 \mathrm{CH}$ bonds. That value was determined using Figure 12 assuming the average PAH contains 50 carbon atoms (Tielens 2008). Table 6 shows the estimates for the percentages of carbon locked in neutral PAHs around YSOs. Sellgren et al. (1995) estimated this value for Mon R2 IRS 3 to be about $4 \%$ which is half of the value reported in this work, but they also used a higher band strength for the $\mathrm{CH}$ bond of $1.7 \times 10^{-18} \mathrm{~cm} /$ bond. With a larger sample of YSOs, Brooke et al. (1999) estimate the percentage of carbon in PAHs to range from $8 \%$ to $23 \%$.

We chose the $\mathrm{CH}$ stretching bands to constrain PAH abundances because they tend to be most consistent across PAH species, but Keane et al. (2001) estimated PAH abundances toward YSOs using excess absorption at $6.2 \mu \mathrm{m}$ instead. Their estimates for the percentage of total carbon locked in PAHs ranged from $83 \%$, assuming neutral $\mathrm{PAHs}$, to $7 \%$, assuming ionized PAHs. This vast difference is due to the fact that neutral PAHs have relatively strong $3.25 \mu \mathrm{m}$ features and weak 

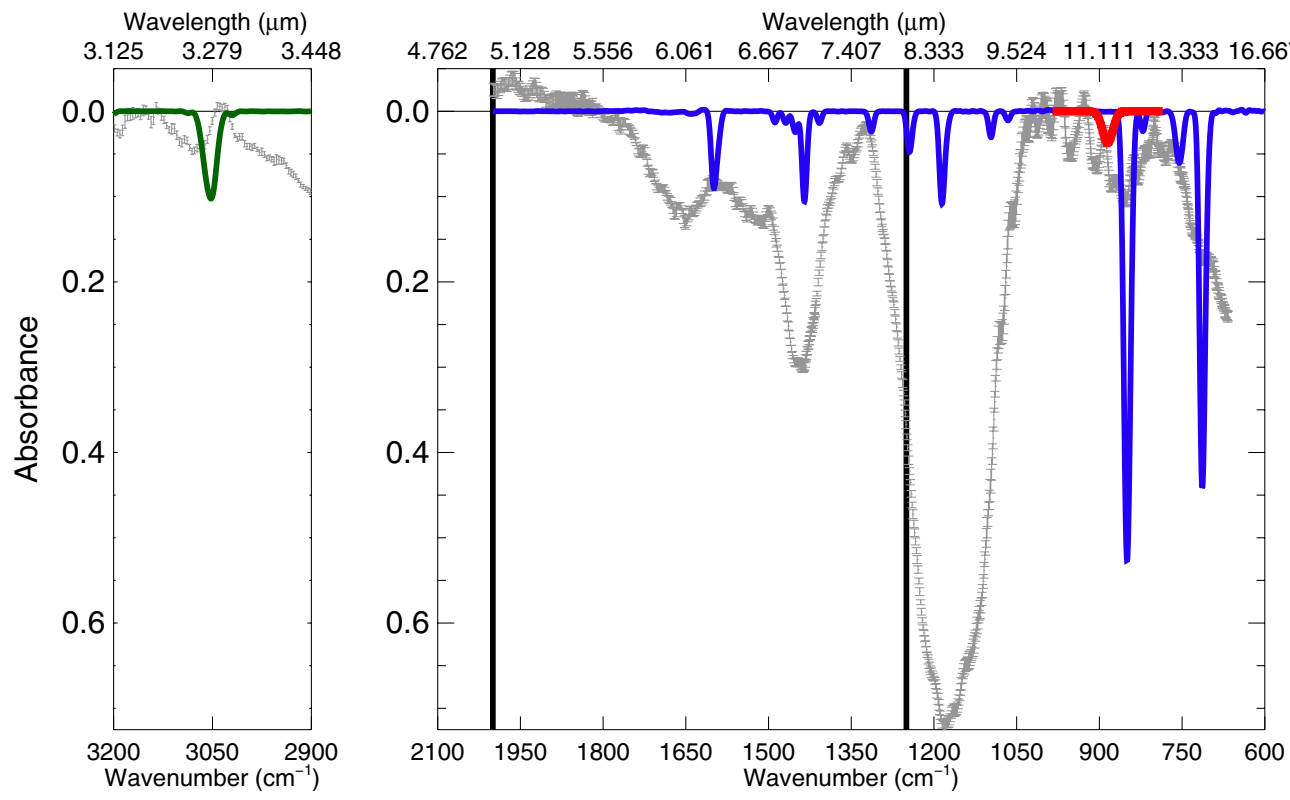

Figure 11. Observed absorption spectrum (gray) of Mon R2 IRS 3 is compared to the laboratory spectrum of pyrene. The left panel shows the residual $3.25 \mu \mathrm{m}$ feature after subtraction of a local baseline (see Section 4.1). The YSO spectrum from 5 to $15 \mu \mathrm{m}$ is plotted after removal of $\mathrm{H}_{2} \mathrm{O}$ ice and silicate absorption (Boogert et al. 2008). The Gaussian at $11.3 \mu \mathrm{m}$ (red) corresponds to the PAH detection reported by Bregman et al. (2000). The pyrene spectrum (green/blue) was scaled so that the area of band A is equivalent to the area of the corresponding YSO feature. All observational and experimental spectra were convolved to a matching resolution of $\sim 8 \mathrm{~cm}^{-1}$. Vertical lines denote the 5-8 $\mu \mathrm{m}$ region where we estimated the PAH contribution to observed absorption.

(A color version of this figure is available in the online journal.)

Table 5

Percent Contribution to the 5-8 $\mu$ m Region $\left(f_{\mathrm{PAH}}\right)$ After $\mathrm{H}_{2} \mathrm{O}$ Subtraction

\begin{tabular}{|c|c|c|c|c|c|}
\hline \multirow[t]{2}{*}{ Object } & \multirow[t]{2}{*}{$N(\mathrm{CH})\left(10^{18} \mathrm{~cm}^{-2}\right)$} & \multicolumn{2}{|c|}{$N(\mathrm{CC})\left(10^{18} \mathrm{~cm}^{-2}\right)$} & \multicolumn{2}{|c|}{$f_{\mathrm{PAH}}(\%)$} \\
\hline & & $\frac{n_{\mathrm{CC}}}{n_{\mathrm{CH}}}=1.375$ & $\frac{n_{\mathrm{CC}}}{n_{\mathrm{CH}}}=4$ & $\frac{n_{\mathrm{CC}}}{n_{\mathrm{CH}}}=1.375$ & $\frac{n_{\mathrm{CC}}}{n_{\mathrm{CH}}}=4$ \\
\hline Mon R2 IRS 3 & $3.76 \pm 0.41$ & $5.17 \pm 0.56$ & $15.03 \pm 1.62$ & 2.9 & 8.5 \\
\hline S140 IRS 1 & $2.05 \pm 0.23$ & $2.81 \pm 0.32$ & $8.18 \pm 0.92$ & 2.0 & 6.0 \\
\hline GL $2136^{\mathrm{a}}$ & $13.34 \pm 2.30$ & $18.34 \pm 3.16$ & $53.36 \pm 9.18$ & 6.9 & 20.4 \\
\hline
\end{tabular}

Notes. ${ }^{a}$ Brooke et al. (1999) report that the inflection point of a crystalline ice absorption profile coincides with the apparent "feature" in GL 2136, so the calculated abundances reported here for this system may be inaccurate.

Table 6

Carbon Budget for Neutral PAHs Around YSOs

\begin{tabular}{lccccc}
\hline \hline Object & $\begin{array}{c}N_{\mathrm{H}^{\mathrm{a}}} \\
\left(10^{22} \mathrm{~cm}^{-2}\right)\end{array}$ & $\begin{array}{c}N_{\mathrm{C}}, \text { total } \\
\left(10^{18} \mathrm{~cm}^{-2}\right)\end{array}$ & $\begin{array}{c}N(\mathrm{CH}) \\
\left(10^{18} \mathrm{~cm}^{-2}\right)\end{array}$ & $\begin{array}{c}N_{\mathrm{C}}, \text { in PAHs } \\
\left(10^{18} \mathrm{~cm}^{-2}\right)\end{array}$ & $\begin{array}{c}\text { \% Carbon } \\
\text { in Neutral PAHs }\end{array}$ \\
\hline Mon R2 IRS 3 & 26.19 & 102.14 & 3.76 & 8.55 & 8.37 \\
S140 IRS 1 & 22.41 & 87.40 & 2.05 & 4.66 & 5.33 \\
GL 2136 & 35.20 & 137.67 & 13.34 & 30.32 & 22.02 \\
\hline
\end{tabular}

Notes.

${ }^{a}$ Reported by Boogert et al. (2013).

b Brooke et al. (1999) report that the inflection point of a crystalline ice absorption profile coincides with the apparent "feature" in GL 2136, so the calculated abundances reported here for this system may be inaccurate.

5-10 $\mu \mathrm{m}$ features, while ionized PAHs exhibit the opposite behavior. According to our estimates, Mon R2 IRS 3 and S140 IRS 1 have less than $10 \%$ of their total carbon in neutral PAHs. These estimates are upper limits in terms of neutral PAH abundances since they assumed the entire $3.25 \mu \mathrm{m}$ features were due solely to neutral PAHs. If the environments near YSOs follow the same trends as the rest of the galaxy, namely that PAHs in these systems contain 10\%-20\% of the carbon reservoir, ionized PAHs should account for the remaining PAH contribution to the carbon budget. When ionized PAHs are accounted for, the portion of absorption in the 5-8 $\mu \mathrm{m}$ spectral region due to
PAHs should be even higher than what is reported in Table 5 . The estimate for GL 2136 that neutral PAHs account for $22 \%$ of its total carbon seems high, lending support to the conclusion of Brooke et al. (1999) that absorption from crystalline $\mathrm{H}_{2} \mathrm{O}$ ice is responsible for the apparent $3.2 \mu \mathrm{m}$ "feature" in its spectrum. Therefore, caution must be exercised when interpreting the cause of absorption in this region.

\section{SUMMARY AND FUTURE WORK}

PAHs account for $10 \%-20 \%$ of the Milky Way's carbon reservoir, yet their direct observation via absorption in the 


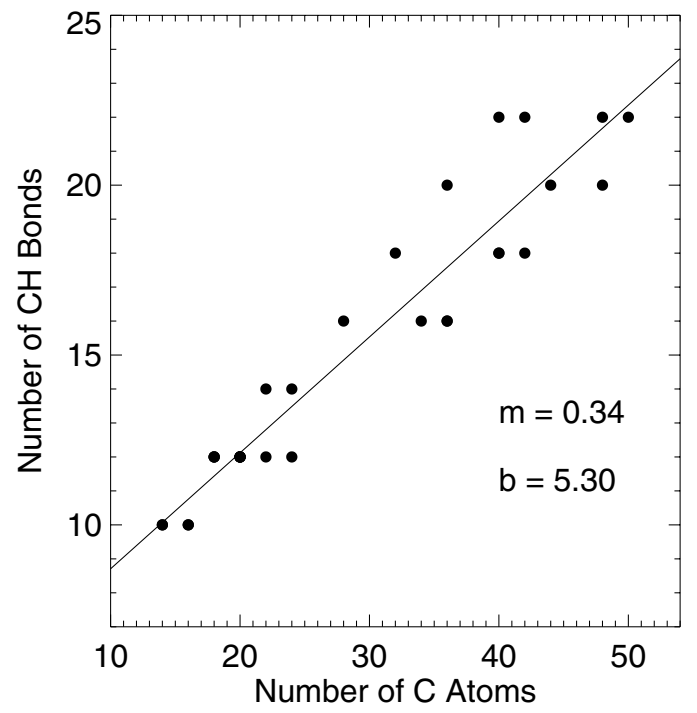

Figure 12. Relationship between number of $\mathrm{CH}$ bonds and number of carbon atoms for PAHs ranging in size from $\mathrm{C}_{14} \mathrm{H}_{10}$ through $\mathrm{C}_{50} \mathrm{H}_{22}$, as listed in the experimental section on www.astrochem.org (Bauschlicher et al. 2010; Boersma et al. 2014). According to this relationship, an average-size PAH containing 50 $\mathrm{C}$ atoms (Tielens 2008) should have roughly $22 \mathrm{CH}$ bonds.

dense ISM remains difficult. Quantification of PAHs expected to be embedded in interstellar ices is hindered by the limited knowledge of the absolute band strengths, peak wavelengths, and band widths for PAHs frozen in ice matrices. Our work addresses this issue via an investigation of pyrene embedded in water ice.

1. We report the first laboratory determination of the band strength for the $\mathrm{CH}$ stretching mode of pyrene in water ice near $3.25 \mu \mathrm{m}$.

2. We report new absolute band strengths from 3 to $15 \mu \mathrm{m}$ for pyrene embedded in $\mathrm{H}_{2} \mathrm{O}$ and $\mathrm{D}_{2} \mathrm{O}$ ice. The band strengths reported here are roughly $50 \%$ greater than those published by Bouwman et al. (2011).

3. We combed the data set of YSO spectra published by Boogert et al. (2008) to look for evidence of PAH absorption at $3.25 \mu \mathrm{m}$ and used the results from our laboratory measurements to estimate PAH column densities where applicable. In our sample, neutral PAHs account for 2\%-9\% of the non- $\mathrm{H}_{2} \mathrm{O}$ ice absorption from 5 to $8 \mu \mathrm{m}$. Neutral PAHs account for 5\%-9\% of the carbon budget toward YSOs, depending on the particular PAH mixture present.

Further laboratory work is needed to determine widths, peak positions, and band strengths for a large variety of PAH species embedded in ices. Figure 11 shows why this is critical. It is obvious from a comparison of the observed absorption of Mon R2 IRS 3 to our laboratory measurements that pyrene alone cannot explain the feature at $3052 \mathrm{~cm}^{-1}(3.25 \mu \mathrm{m})$ attributed to $\mathrm{CH}$ stretching. Band $\mathrm{A}$ in our laboratory spectra is too narrow and slightly offset from the observed $3.25 \mu \mathrm{m}$ feature. Beyond $10 \mu \mathrm{m}$, the $\mathrm{CH}$ out of plane bending features for pyrene do not align with the PAH detection at $11.3 \mu \mathrm{m}$ reported by Bregman et al. (2000). In addition, the pyrene features in this region are much stronger than the reported $11.3 \mu \mathrm{m}$ feature. This apparent discrepancy supports the conclusion that PAHs around Mon R2 IRS 3 must contain a mixture of species. As illustrated in Figure 2 of Allamandola et al. (1999), a mixture of neutral PAHs will exhibit a strong, narrow feature at $3.25 \mu \mathrm{m}$ and much broader, weaker features beyond $10 \mu \mathrm{m}$. This is because the position of the $\mathrm{CH}$ stretching mode is quite stable across $\mathrm{PAH}$ species whereas the $\mathrm{CH}$ out of plane bending modes, though stronger for any individual species, are much more variable in position. The result is an apparent broadening and weakening of the composite absorption feature beyond $10 \mu \mathrm{m}$ relative to the $3.25 \mu \mathrm{m}$ feature in the spectrum of a PAH mixture.

Future work should also investigate the effects of other relevant ice matrices (e.g., $\mathrm{CO}_{2}$ ) on the $\mathrm{PAH}$ absorption profiles. Eventually, the infrared band strengths for a variety of ionized PAHs need to be measured to compliment work with neutral species. Finally, the greatest difficulty for detecting PAH absorption features in YSO spectra is the lack of sufficient signalto-noise due to atmospheric effects (in particular telluric $\mathrm{CH}_{4}$ lines) in the ground-based data. Observational campaigns during the upcoming SOFIA and James Webb Space Telescope missions should effectively solve this problem.

This research was carried out at the Jet Propulsion Laboratory and IPAC, California Institute of Technology, under a contract with the National Aeronautics and Space Administration. Support for this research was provided in part by funding from the NASA Astrobiology Institute to Rensselaer Polytechnic Institute (award NNA09DA80A). The experimental part of this work was enabled through partial funding from the Jet Propulsion Laboratory's DRDF and R\&TD funding for infrastructure of the "Ice Spectroscopy Laboratory" and was carried out by H.L. and M.S.G., supported by an astrophysics laboratory research award funded by the Spitzer Space Telescope. H.L. also acknowledges the Finnish Cultural Foundation for financial support. We thank Dr. Irene Li Barnett and Dr. Antti Lignell, who were involved in building the experimental setup used and initial training of H.L. Data analysis was carried out by E.H-U. at the California Institute of Technology (supervised by A.B. and M.S.G.), funded by an IPAC Visiting Graduate Student Fellowship.

\section{REFERENCES}

Allamandola, L. J. 2011, in EAS Publications Series, Vol. 46, EAS Publications Series, ed. C. Joblin \& A. G. G. M. Tielens, 305

Allamandola, L. J., Hudgins, D. M., \& Sandford, S. A. 1999, ApJL, 511, L115 Allamandola, L. J., Sandford, S. A., Tielens, A. G. G. M., \& Herbst, T. M. 1992, ApJ, 399, 134

Barnett, I. L., Lignell, A., \& Gudipati, M. S. 2012, ApJ, 747, 13

Bauschlicher, C. W., Jr. Boersma, C., Ricca, A., et al. 2010, ApJS, 189, 341

Bauschlicher, C. W., Jr. Peeters, E., \& Allamandola, L. J. 2008, ApJ, 678, 316

Berlman, I. B. 1971, Handbook of Fluorescence Spectra of Aromatic Molecules (2nd ed.; New York: Academic)

Bernstein, M. P., Dworkin, J. P., Sandford, S. A., Cooper, G. W., \& Allamandola, L. J. 2002, Natur, 416, 401

Bernstein, M. P., Sandford, S. A., \& Allamandola, L. J. 2005, ApJS, 161, 53

Bernstein, M. P., Sandford, S. A., Mattioda, A. L., \& Allamandola, L. J. 2007, ApJ, 664, 1264

Boersma, C., Bauschlicher, C. W., Ricca, A., et al. 2014, ApJS, 211, 8

Boogert, A. C. A., Chiar, J. E., Knez, C., et al. 2013, ApJ, 777, 73

Boogert, A. C. A., Pontoppidan, K. M., Knez, C., et al. 2008, ApJ, 678, 985

Bouwman, J., Mattioda, A. L., Linnartz, H., \& Allamandola, L. J. 2011, A\&A, 525, A93

Bowman, K. P. 2006, An Introduction to Programming with IDL: Interactive Data Language (New York: Academic)

Bregman, J. D., Hayward, T. L., \& Sloan, G. C. 2000, ApJL, 544, L75

Brooke, T. Y., Sellgren, K., \& Geballe, T. R. 1999, ApJ, 517, 883

Brooke, T. Y., Sellgren, K., \& Smith, R. G. 1996, ApJ, 459, 209

Dartois, E., \& d'Hendecourt, L. 2001, A\&A, 365, 144

Dixon, J. M., Taniguchi, M., \& Lindsey, J. S. 2005, Photochem. Photobiol., 81, 212

Geers, V. C., van Dishoeck, E. F., Pontoppidan, K. M., et al. 2009, A\&A, 495,837

Gibb, E. L., Whittet, D. C. B., Boogert, A. C. A., \& Tielens, A. G. G. M. 2004, ApJS, 151, 35 
Gudipati, M. S. 2004, JPCA, 108, 4412

Gudipati, M. S., \& Allamandola, L. J. 2003, ApJL, 596, L195

Gudipati, M. S., \& Allamandola, L. J. 2004, ApJL, 615, L177

Gudipati, M. S., \& Allamandola, L. J. 2006, ApJ, 638, 286

Gudipati, M. S., \& Yang, R. 2012, ApJL, 756, L24

Kaiser, R. I., Stockton, A. M., Kim, Y. S., Jensen, E. C., \& Mathies, R. A. 2013, ApJ, 765, 111

Keane, J. V., Tielens, A. G. G. M., Boogert, A. C. A., Schutte, W. A., \& Whittet, D. C. B. 2001, A\&A, 376, 254

Markwardt, C. B. 2009, in Astronomical Society of the Pacific Conference Series, Vol. 411, Astronomical Data Analysis Software and Systems XVIII, ed. D. A. Bohlender, D. Durand, \& P. Dowler (San Francisco, CA: ASP), 251

Muñoz Caro, G. M., Meierhenrich, U. J., Schutte, W. A., et al. 2002, Natur, 416, 403
Peeters, E. 2011, in IAU Symp. 280, The Molecular Universe, ed. J. Cernicharo \& R. Bachiller (Cambridge: Cambridge Univ. Press), 149

Ray, G. B., Chakraborty, I., \& Moulick, S. P. 2006, J. Colloid Interface Sci., 294, 248

Salama, F., \& Allamandola, L. J. 1992, Natur, 358, 42

Sandford, S. A., Bernstein, M. P., \& Allamandola, L. J. 2004, ApJ, 607, 346

Sellgren, K., Brooke, T. Y., Smith, R. G., \& Geballe, T. R. 1995, ApJL, 449, L69

Sellgren, K., Smith, R. G., \& Brooke, T. Y. 1994, ApJ, 433, 179

Siu, H., \& Duhamel, J. 2008, JPCB, 112, 15301

Thony, A., \& Rossi, M. J. 1997, J. Photochem. Photobiol. A-Chem., 104, 25

Tielens, A. G. G. M. 2008, ARA\&A, 46, 289

Whittet, D. C. B., (ed.) 2003, Dust in the Galactic Environment (Bristol: Institute of Physics)

Williams, M. J., Bureau, M., \& Cappellari, M. 2010, MNRAS, 409, 1330 